\title{
LOS ROBLEDALES GALAICO-SEPTENTRIONALES
}

\author{
Jesús IZCO, Javier AMIGO \& Javier GUITIAN
}

\begin{abstract}
RESUMEN: Se estudian los robledales galaico-septentrionales que se incluyen en la asociación Blechno spicantis-Quercetum roboris Tx.\& Oberd. 1958, describiéndose la nueva subasociación pulmonarietosum longifoliae. Se aportan datos sobre su serie de vegetación y sus relaciones con otros robledales afines.
\end{abstract}

Palabras clave: Bosque atlántico, Bosque caducifolio, Robledales, Provincia CántabroAtlántica.

SUMMARY: The oakwoods of northern Galicia, included in the association Blechno spicantis-Quercetum roboris are studied; the pulmonarietosum longifoliae subassociation has been described. Several data on these particular woods are also reported, including the vegetation serie and the relationships with other similar vegetation types.

Key words: Decidous forest, west-european atlantic forest, Quercus robur forest, CantabroAtlantic province.

\section{N T R O D U C C I O N}

Aunque el nivel de conocimiento de la vegetación del occidente ibérico ha mejorado sustancialmente en los últimos años, existen todavía aspectos puntuales que precisan un estudio más profundo. En esta línea, ha resultado tradicionalmente conflictiva la delimitación entre las distintas unidades corológicas en el norte de Galicia -sector Galaico-Asturiano- debido tanto a la falta de datos como a que se trata de territorios muy alterados.

El presente trabajo trata de aportar información sobre los robledales galaicoseptentrionales y su serie de vegetación, así como de facilitar su discriminación de otros robledales afines.

\section{A N T E C ED E N T E S}

Los primeros datos sobre estos robledales fueron dados a conocer por Tüxen y Oberdorfer (1958) quienes describen la asociación Blechno-Quercetum roboris basada en seis inventarios procedentes de Galicia y Asturias (ver su tabla $\mathrm{n}^{\mathrm{o}} 76$ ). 
Se trata de una tabla heterogénea pero que, en nuestra opinión, permite reconocer suficientemente la composición florística y variabilidad de estos robledales. Con posterioridad Bellot (1968) incluye en su tabla del Quercetum roboris gallaecicum algunos inventarios referibles a la asociación Blechno-Quercetum roboris (inv. 16 y 17) junto a otros, la gran mayoría, referibles a la asociación más meridional Rusco-Quercetum roboris.

Losa Quintana (1973) a través de un estudio de los robledales de la cuenca media del río Eume, da a conocer dos subasociaciones del Blechno-Quercetum, la primera, coryletosum avellanae de carácter más húmedo, y la segunda lauretosum nobilis de carácter marcadamente más termófilo.

Los inventarios de T. Díaz (1975) del Occidente de Asturias y los comentarios de Rivas-Martínez \& al. (1984) completan la información existente sobre estos bosques.

\section{Blechno spicantis-Quercetum roboris Tüxen \& Oberdofer 1958.}

Tipificación: Fijamos el tipo de la asociación en el inventario 136 de la tabla original (Tabla 76, Tx. \& Oberd., op, cit.) realizado por Oberdorfer entre Ribadeo y Lugo (Sierra de Meira), correspondiente a la "subasociación con Dryopteris aemula". Aunque su ubicación geográfica no es excesivamente precisa, parece lo más apropiado al tratarse del único inventario en que Castanea sativa no es el árbol dominante.

Estructura y Ecología: Bosques de Quercus robur estructurados en tres estratos con elevada cobertura y un número de especies por inventario próximo a 30 . En el estrato arbóreo el roble comparte, o cede en casos, su dominio con Castanea sativa, no faltando Betula celtiberica y Sorbus aucuparia.

Desde el punto de vista edáfico se desarrollan en suelos con marcado carácter ácido, en general, cambisoles originados a partir de distintos materiales (esquistos, pizarras, granitos, etc.).

Composición florística y variabilidad: Se reconocen tres subasociaciones:

a) subas. dryopteridetosum aemulae (Tabla 1, inv. 1-22).

Es la que representa el aspecto típico de la comunidad y corresponde a la "subasociación con Dryopteris aemula" de Tüxen y Oberdorfer (op. cit.). Se caracteriza florísticamente por la presencia de Betula celtiberica, Vaccinium myrtillus, Dryopteris aemula, Dryopteris dilatata, Polygonatum verticillatum, Ranunculus nemorosus y Sorbus aucuparia. Desde el punto de vista bioclimático se encuadra óptimamente en el horizonte eucolino.

A ella corresponden los dos inventarios del Quercetum roboris gallaecicum antes mencionados.

b) subas. lauretosum nobilis Losa Quintana ex Izco, Amigo \& Guitián (Tabla 1, inv. 23-32. Tipo inv. 28).

Caracterizada por la presencia de elementos termófilos como Laurus nobilis, Arbutus unedo, Rubia peregrina, Ruscus aculeatus y Smilax aspera, tiene su óp- 



\begin{tabular}{|c|}
\hline 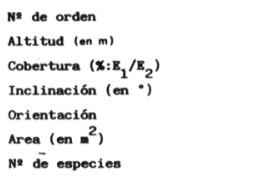 \\
\hline Características Ass, Al. et Or. \\
\hline Quercus nobur \\
\hline Holcus mollis \\
\hline Blechrum spicant \\
\hline Vacciniem mertilles \\
\hline Betula celtiberica \\
\hline Teucrium scorodonia \\
\hline Pyrus pyraster \\
\hline Castanea sativa \\
\hline Physospernum connubiense \\
\hline Soxifraga spathularis \\
\hline Melompyrum pratense \\
\hline Polygonatum verticillatum \\
\hline Omphalodes nitida \\
\hline Hypericum pulchum \\
\hline amunda regalis \\
\hline Luzula henriquesii \\
\hline Oryopteris aemula \\
\hline
\end{tabular}

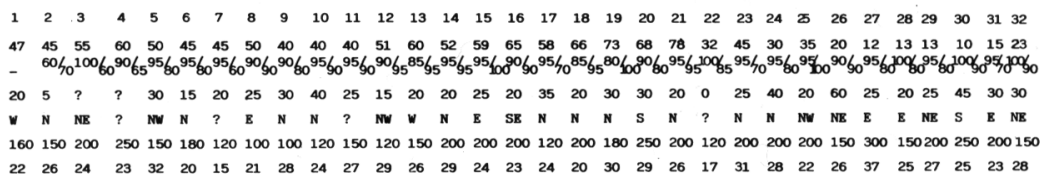

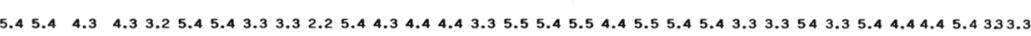

$1.11 .12 .21 .22 .1+$ + $1.11 .11 .11 .11 .12 .22 .2+.2+2.22 .22 .22 .1++1.11 .1 \cdot 2.21 .1++2.1+2+$ $2.22 .2 \cdot \int 2.21 .11 .11 .11 .11 .11 .11 .22 .21 .12 .21 .21 .22 .21 .1+1.1+1.12 .2221 .12 .23 .33 .3$ + +1.2 $2.22 .2 \quad 2.3 \quad 3.42 .33 .22 .23 .32 .23 .34 .44 .32 .32 .34 .34 .41 .24 .42 .23 .24 .34 .42 .31 .154$. 1.1 . $\quad . \quad$. 1.23 .2

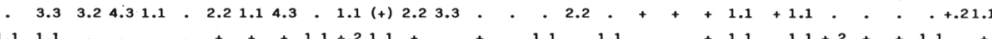

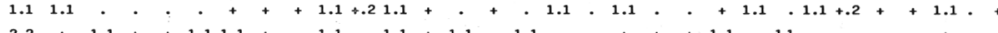

$2.2+1.1++1.11 .1+\cdot 1.1 \cdot 1.1+1.1 \cdot 1.1 \cdot \cdot+++1.1 \cdot 11 \cdot \cdot \cdot++\cdot$

. $1.11 .1 .+1.12 .2+. .2 .1$. .1 .1 . . . . . + . . $2.2+3.32 .22 .22 .2+1.13 .3$ $++\cdot+\cdot \cdot+\cdot r+\cdot+\cdot \cdot+.2 \cdot \cdot+\cdot r+\cdot \cdot \cdot+1.1++1.11 .1$

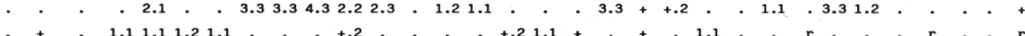

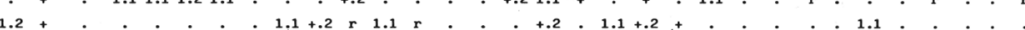

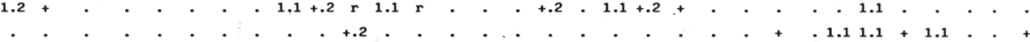

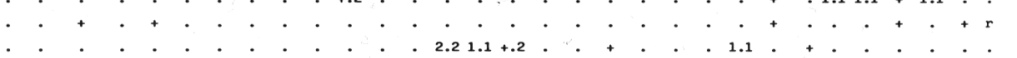
$1.3++.3$

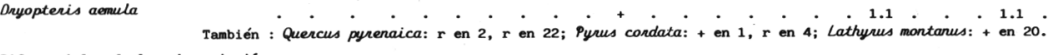

Diferenciales de la subasociación

Ruscus aculeatus

Anbutus unedo

Launus nobilis

Rubia peregrina ct. longifolia

Tambien : Aspleniwm onopteris: + en 30 ; smilax aspera: + en 31.

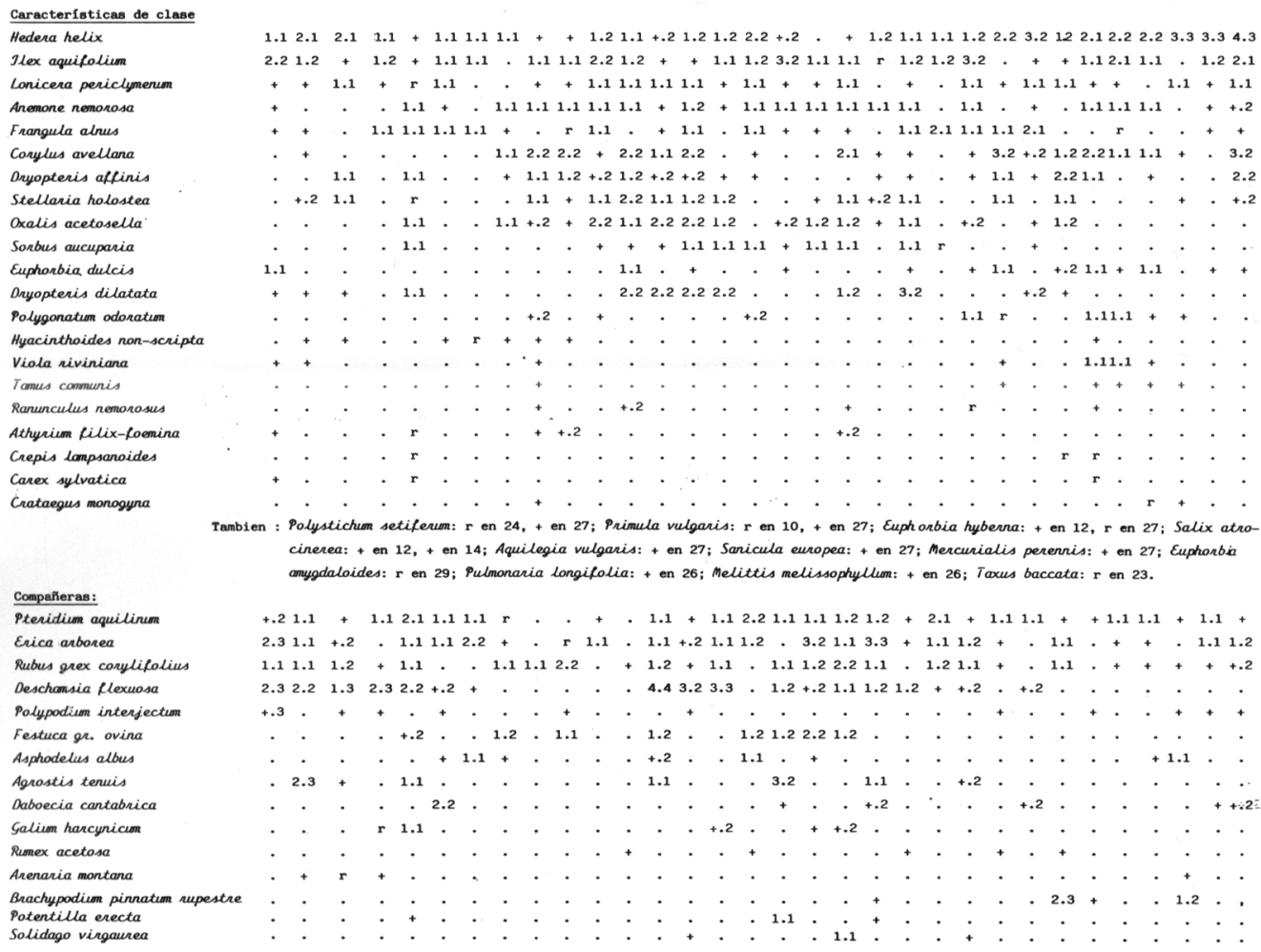

También : Ulex europaeus: + en 4, + en 22; Avenula sulcata: +.2 en 4, + en 17; Pseudanhenaterum Longifolium: 1.1 en 20, $r$ en 22; Lastraea limbosperma: $\mathrm{r}$ en 13, 1.1 en 19; Prunus laurocerassus: + en 3; Anthoxanthum omarum: + en 3; Dactylonhhiza incarnata: en 4; Anthoxanthum odoratum: + en 24; Peucedanum laucifolium: + en 30; Senecio nemonersis subsp. bayonensis: $r$ en 12; Ajuga reptans: + en 12; Agrostis curtisii: 2.3 en 17; Simetis mattiazzi: 1.1 en 17; Conopodium of.pyrenaeum: + en 19; Cruciata lae reptans: + en 12; Agrostis curtisi: 2.3 en 17; Simetis mattiazzi: 1.1 en 17; Conop

LOCALIDADES:

1/. C - Monfero; montes de Serra da Loba entre Irixoa y el Ifmite provincial (29TNH 8697). 2/ C - Monfero; c. del anterior en otra ladera (29TNH 8798). 3/6 Lu Muras; bajando al pueblo desde Xermade (29TPJ 0412). 4/ Lu - Muras; saliendo hacia Viveiró (29TPJ 0315). 5/ Lu - Ourol; ctra. de Muras hacia Sisto, bajando al Oeste hacia Ambosores (29TPJ 0422). 6/ C - Capela; embalse del Eume, ladera Norte (29TNJ 8007). 7 / C - Monfero; Fraga de Pontedapena, c. Xestoso (29TNJ 8903). 8/ C - As Pontes de Ge Rodriguez; bosque de Adá entre As Pontes y Deveso (29TNJ 9420). 9/ C - Somozas; entre Somozas y As Pontes, c. Recemel (29TNJ 8515). 10/ C - Somozas; al lado del anteror (29TNJ 8516). 116 Lu - Valadouro; ctra. Ferreira-Viveiro, c. Lobeiras (29TPJ 2224). 12/ Lu - Trabada; Vilapena (29TPJ 4307). 13/ Lu - Riotorto; Augaxosa (29TPJ 4207). 14/ Lu - Trabada; fraga en Serra da Cadeira (29TPJ 4309). 15/. O - Taramundi; entre Bres y Sta. Eulalia de Oscos, c. Paramios (29TPJ 6004). 16/ Lu - Ribeira de Piquín; entre Couso y Vilar de Corota (29TPH 4386). 17/ Lu Mondon̂edo; Estelo (29TPJ 2310). 18/ Lu - Muras; Aborbb, entre A Balsa y Viveirb (29TPJ 1010). 19/ Lu - Muras; de Viveirb hacia Muras (29TPJ 1014 ). 206 Lu - Mondoñedo; Fraga Vella (29TPJ 2912). 21/ Lu - A Pontenova; alto de la devesa de Sanxés, cabecera del río Bidueiro (29TPH 5394). 22/ Lu - Ourol; Xerdiz. c. del rio Xuanceda (29TPJ 1122). 23/ Lu - Cervo; monte de RGa en la cabecera del río Xunco (29TPJ 2628). 24/6 C - Capela; junto a la central del embalse del Eume (29TNJ 7806). 25/ Lu - Trabada; Meixoedo (29TPJ 4610). 26/ Lu - Ourol; Segade, en las laderas del río Sor (29TPJ 0230). 27/ C - Cerdido; devesa del Mera, c. Casares (29TNJ 8826). 28 / C - Cerdido; pr6ximo al anterior (29T NJ 8826). 29/ C - Cerdido; mismo bosque que los anteriores (29TNJ 8826). 30/ C - Capela; Fraga de Caaveiro entre el monasterio y la Central (29TNJ 7607). 31/ Lu - Trabada; S. Román (29TPJ 4912). 32/ Lu Ribadeo; Arante (29TPJ 4616). 

timo en el horizonte termocolino; sin embargo, parece ser que hacia oriente y en concreto en el subsector Ovetense, es frecuente que rebase los niveles altitudinales de este horizonte, ya que estos táxones termófilos irrumpen sin dificultades en los niveles eucolinos.

A esta subasociación corresponde la propuesta inválida de Losa Quintana (op. cit.) ya que es imposible determinar qué inventarios pertenecen a la subasociación; las especies que da como diferenciales, al igual que las de otras subasociaciones propuestas en el mismo trabajo, son comunes a la práctica totalidad de los inventarios; además los grupos resultantes del análisis matemático no coinciden con los por él enunciados en el capítulo sintaxonómico.

c) subas. pulmonarietosum longifoliae nova (Tabla 2, inv. 1-3).

La presencia de un mayor contingente de elementos de Fagetalia permite independizar esta nueva subasociación para la que proponemos como tipo el inventario no 2 de la tabla 2. Son diferenciales de esta subasociación Fraxinus excelsior, Ulmus glabra, Sanicula europea, Primula vulgaris y Pulmonaria longifolia. Desde el punto de vista topográfico ocupa posiciones de vaguada o de fondo de valle de mayor trofía y frescura de suelo.

Esta subasociación representa el biotopo adecuado para la pervivencia de táxones eutrofos y humícolas que en la Asturias centro-oriental se integran en las formaciones de Carpinion betuli. El cambio geológico a los sustratos masivamente ácidos en Asturias occidental y norte de Galicia, impide la presencia del Carpinion en el piso colino. Por ello, las plantas de óptimo en este sintaxon que alcanzan el occidente, se integran en la aliseda (Valeriano pyrenaicae-Alnetum glutinosae) o bien en estas "'carballeiras"" de vaguada.

En la Tabla 3 hemos recogido 4 inventarios de lo que denominamos "facies de Castanea sativa"'", que en nuestra opinión pueden representar una alternativa de interés para la repoblación de los territorios Galaico-septentrionales en aquellos casos en que no existan limitaciones edáficas, fundamentalmente referidas a profundidad de suelo.

Esta facies es de algún modo convergente con la "subasociación con Hieracium umbellatum" de Tüxen y Oberdorfer, ya que en ambos casos el dominio del estrato superior obliga a hablar de bosques de castaños más que de robles. No obstante, la subasociación con Hieracium umbellatum presenta algunos matices de composición florística (ausencia de táxones noroccidentales como Omphalodes nitida y Saxifraga spathularis) y de procedencia geográfica (subsector Ovetense) que nos permiten matizarla como "raza oriental" frente a los inventarios que nosotros presentamos, y por tanto parece prudente no identificarlos bajo el mismo concepto.

\section{I N A M IS M O}

La serie de vegetación de los robledales Galaico-septentrionales permanecía insuficientemente conocida. Inicialmente parece necesario realizar algunas consideraciones sobre lo recientemente publicado por M.P.A. Castroviejo (1988) referente al dinamismo de estos bosques en la Serra do Xistral. En nuestra opinión, nada tienen que ver los cultivos de Pinus sp. con la sucesión natural en nuestro ámbito geográfico. La serie de vegetación de estos robledales transcurre a través de 
las etapas de piornal, matorral, etc., pero en ningún caso estos cultivos pueden constituir una etapa del dinamismo natural hacia las "carballeiras"; en este sentido, la afirmación de que "Pinus pinaster prepara la entrada de Quercus robur y que por lo tanto debe considerarse como una etapa intermedia previa a la clímax" carece de fundamento.

En lo que se refiere a las etapas naturales de sustitución son piornales y brezales las que presentan mayor interés. Los primeros, que orlan las "carballeiras" desarrollándose sobre suelos de tipo forestal, pueden referirse a dos asociaciones: la Ulici europaei-Cytisetum ingramii (Tabla 4) cuya personalidad florística se basa en la presencia del piorno endémico Cytisus ingramii, se limita a los territorios más occidentales del sector Galaico-Asturiano, rebasando sólo de forma accidental los límites de la provincia de La Coruña; en algún caso (Alvarez \& Horjales, 1977) se ha señalado la relación entre la distribución de Cytisus ingramii y los sustratos ultrabásicos del noroccidente de Galicia. La segunda, Ulici europaei-Cytisetum striati, común a los territorios galaico-portugueses, ocupa posiciones análogas a la anterior, fundamentalmente en los territorios a oriente del río Sor.

La asociación Cytisetum scopario-striati que en algún caso (Díaz y Prieto, 1987) se ha invocado como el sintaxon que englobaría los piornales de esta serie en Asturias occidental, debe precisarse desde el punto de vista florístico.

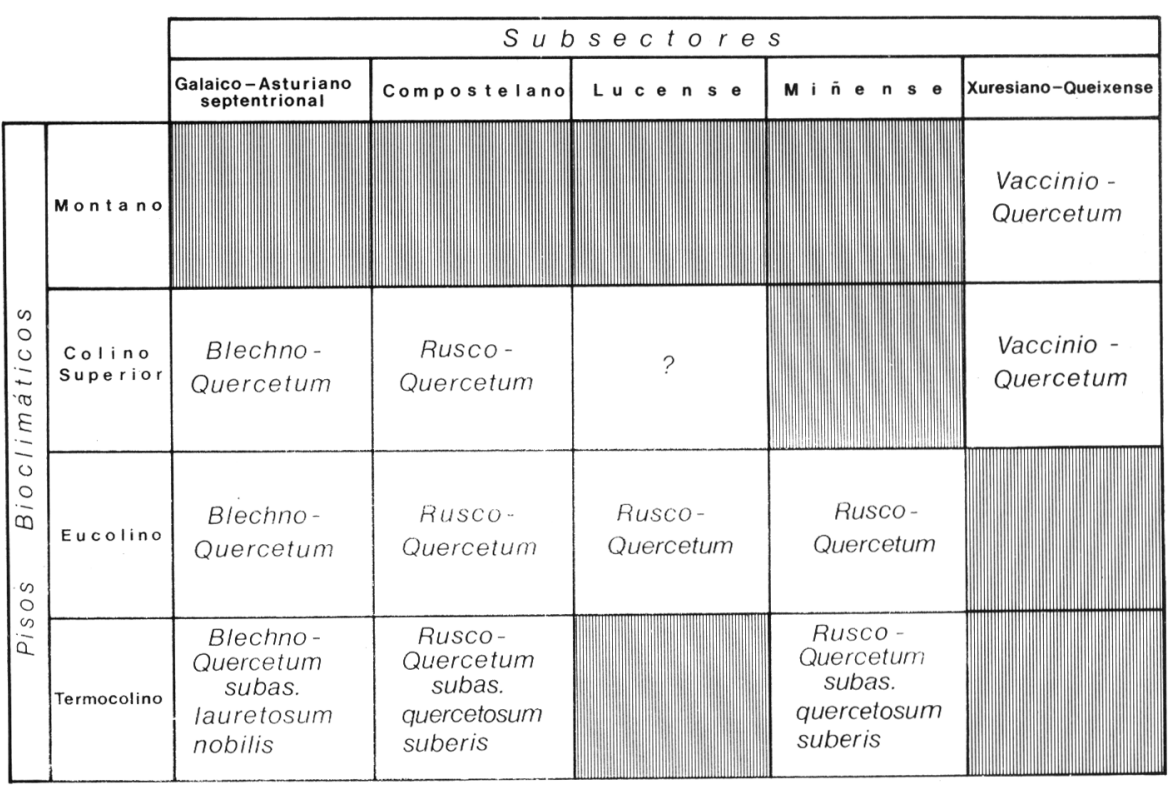

Cuadro 2.- Ordenación corológica y bioclimática de los robledales galaicos de Quercus robur. 
Los brezales de la serie pueden incluirse también en dos asociaciones bien conocidas ya desde el punto de vista florístico: Gentiano pneumonanthe-Ericetum mackaianae, sobre suelos con un mayor grado de podsolización, y Ulici europaeiEricetum cinereae que se desarrolla sobre suelos esqueléticos y en general en todos los enclaves graníticos del territorio.

\section{RELACIONES CON LOS ROBLEDALES AFINES}

El cuadro I recoge de forma sintética las principales relaciones florísticas de los Blechno-Quercetum roboris con los robledales colinos y montanos galaicoportugueses, Rusco-Quercetum roboris y Vaccinio-Quercetum roboris respectivamente, cuyas adscripciones corológicas y bioclimáticas se reflejan en el cuadro II.

Los robledales de Blechno-Quercetum roboris pueden diferenciarse bien de los citados por la presencia de Polygonatum verticillatum, Ranunculus nemorosus y Sorbus aucuparia , así como por la constancia de un buen número de pteridófitos como Dryopteris dilatata, Dryopteris aemula, Athyrium filix-foemina, etc.

En la geoserie, la diferenciación es posible a través de sus contactos respectivos con los bosques ribereños: Valeriano pyrenaicae-Alnetum en el subsector Galaico-septentrional y Senecio bayonensis-Alnetum en el sector Galaico-Portugués.

\section{A P E N D I C E F L OR IS T I C O}

Relación de táxones citados en el texto o en las tablas, en cuya nomenclatura no seguimos el criterio de Tutin \& al. (1964-1980):

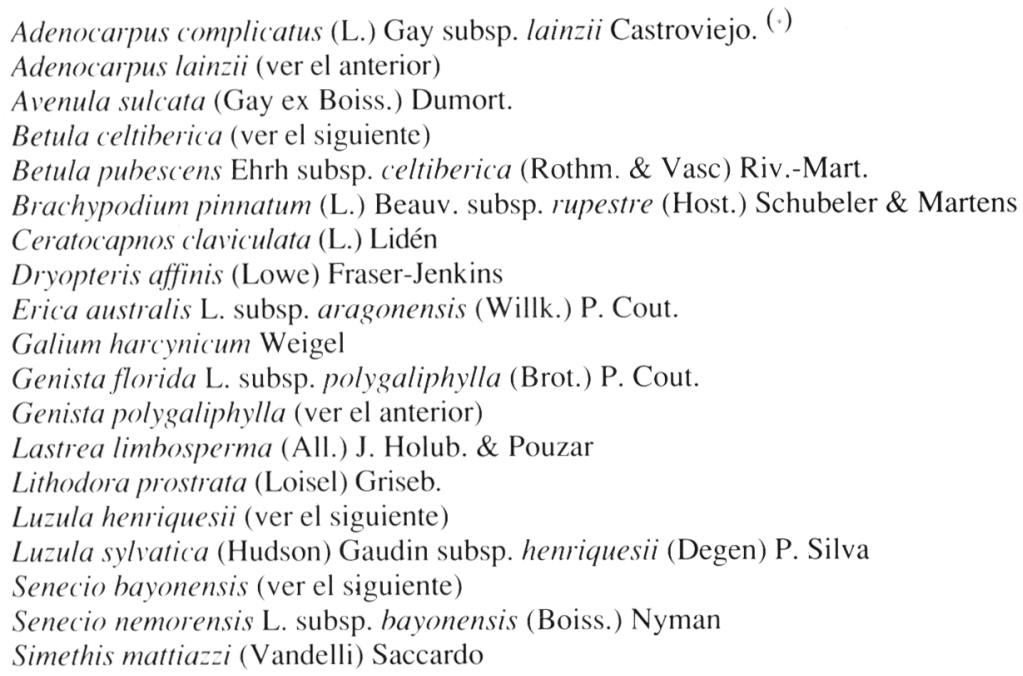

${ }^{*}$ ) Según se pone en claro en reciente revisión de este género (cf. Rivas-Martínez \& Belmonte, 1989) el nombre correcto para este taxon sería A. complicatus subsp. anisochilus (Boiss.) Franco. 


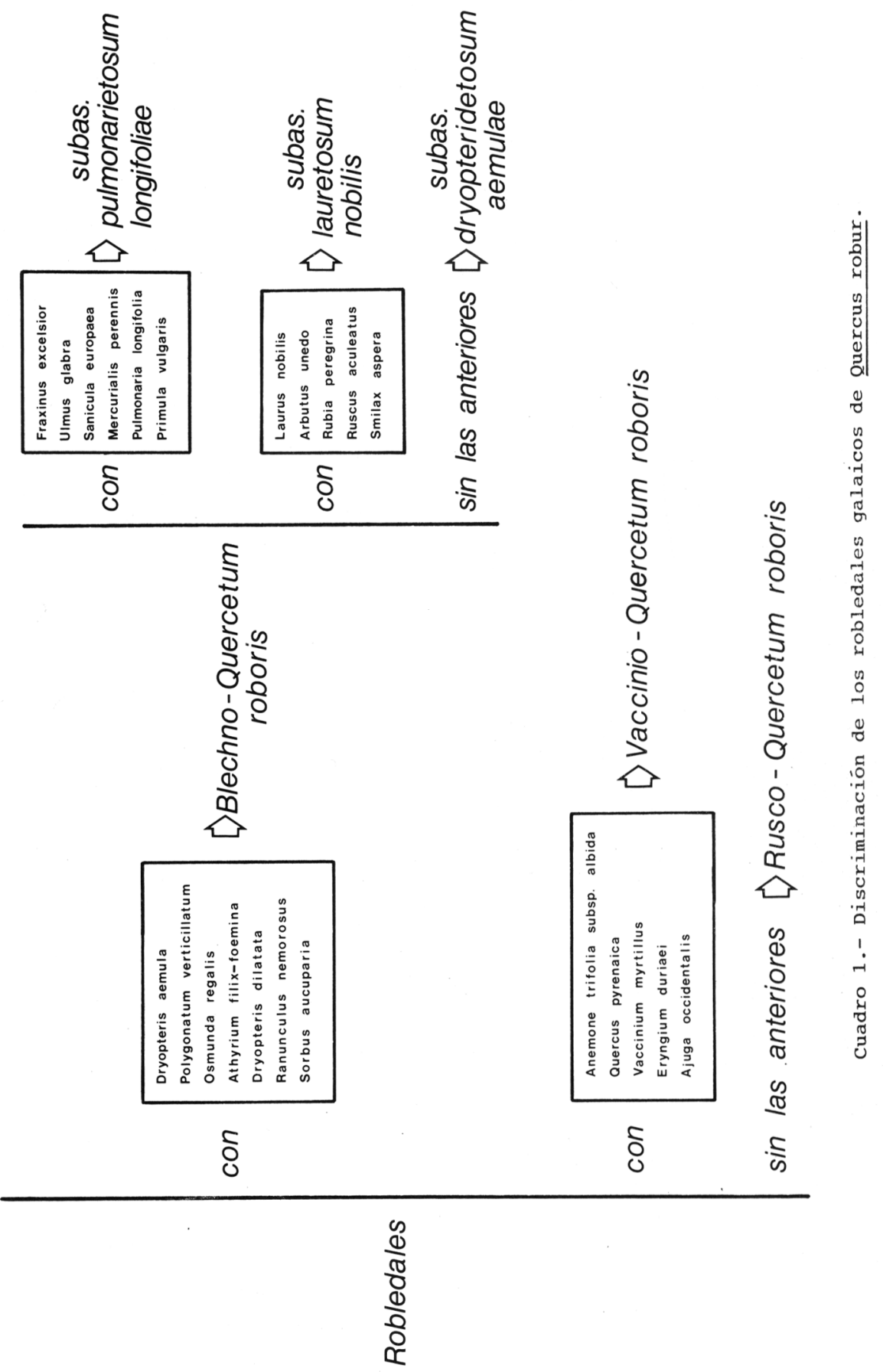




\section{B I B L I O G R A F I A}

ALVAREZ, R. \& HORJALES, M. -1977-Contribución a la corología de Cytisus ingramii Blakelock. Lagascalia 7(1):3-8.

BELLOT, F. - 1968- La vegetación de Galicia. Anal. Inst. Bot. Cavanilles, 24:3-306.

CASTROVIEJO, M.P.A. -1988- Fitoecología de los montes de Buio y Sierra del Xistral (Lugo). Consellería de Agricultura. Xunta de Galicia.

DIAZ, T.E. -1975- La vegetación del litoral occidental asturiano. Rev. Fac. Ciencias. Oviedo, 16:369-545.

DIAZ, T.E. \& PRIETO, J.A.F. -1987- Asturias y Cantabria (en La Vegetación de España, M. Peinado \& S. Rivas-Martínez Eds.). Universidad de Alcalá de Henares.

LOSA QUINTANA, J.M. -1973- Estudio de las comunidades arbóreas naturales de la cuenca media del río Eume. Trab. Compostelanos de Biología, 3:3-63.

RIVAS MARTINEZ, S. \& BELMONTE, D. -Sinopsis de Adenocarpus DC. (Leguminosae). Opusc. Bot. Pharm. Complutensis, 5:69-78.

RIVAS MARTINEZ, S., DIAZ, T.E., FERNANDEZ PRIETO, J.A., LOIDI, J. \& PENAS, A. - 1984- La vegetación de la alta montaña cantábrica. Los Picos de Europa. Ediciones Leonesas, $300 \mathrm{pp}$.

TÜXEN, R. \& OBERDORFER, E. -1958- Die Pflanzenwelt Spaniens, II Teil. Eurosibirische Phanerogamen-Gesellschaften Spaniens. Veröff. Geobot. Inst. Rubel Zurich, 32.

(Aceptado para su publicación el 15 de noviembre de 1989)

Dirección de los autores: Laboratorio de Botánica. Departamento de Biología Vegetal. Facultad de Farmacia. 15706, Santiago de Compostela. 
TABLA 2

Blechno spicantis-Quercetum roboris subas. pulmonarietosum longifoliae

$\begin{array}{lccc}\text { № de orden } & 1 & 2 & 3 \\ \text { Altitud (en Dm) } & 48 & 13 & 40 \\ \text { Cobertura (\%E1) } & 100 & 100 & 100 \\ \text { Cobertura (\%E2) } & 95 & 90 & 75 \\ \text { Inclinación } & 40^{\circ} & 35^{\circ} & 30^{\circ} \\ \text { Orientación } & \mathrm{SW} & \mathrm{E} & \mathrm{N} \\ \text { Area (en m2) } & 60 & 100 & 120 \\ \text { № de especies } & 27 & 38 & 38\end{array}$

Características de Ass., Al., Or., Cl

Corylus avellana

Quercus robur

Blechnum spicant

Dryopteris affinis

Polygonatum verticilatum

Euphorbia dulcis

Anemone nemorosa

Oxalis acetosella

Hedera helix

Athyrium filix-foemina

Brachypodium sylvaticum

Omphalodes nitida

Vaccinium myrtillus

Stellaria holostea

Dryopteris dilatata

Ilex aquifolium

Ruscus aculeatus

Holcus mollis

Lonicera periclymenum

Viola riviniana

Diferenciales de Fagetalia

Primula vulgaris

Ranunculus nemorosus

Pulmonaria longifolia

Sanicula europea

Fraxinus excelsior

Mercurialis perennis

\section{Compañeras}

Rubus gr.corylifolius

Hieracium sp.

$\begin{array}{ccc}5.4 & 5.4 & 2.2 \\ 1.1 & \mathrm{r} & 4.3 \\ 1.1 & 1.1 & 1.1 \\ 2.2 & 1.1 & 1.1 \\ 1.1 & + & \mathrm{r} \\ 1.1 & 1.1 & 1.1 \\ 1.1 & 2.1 & + \\ 1.2 & + & 3.3 \\ +.2 & 1.1 & +.2 \\ 1.2 & 1.1 & 1.1 \\ 3.3 & + & \cdot \\ 1.1 & + & \cdot \\ 1.2 & + & \cdot \\ 1.1 & . & 1.1 \\ + & . & + \\ . & 1.1 & 1.2 \\ \cdot & + & +.2 \\ . & + & + \\ . & + & + \\ . & + & + \\ & & \\ 2.1 & 2.1 & + \\ 1.1 & 1.1 & + \\ 1.1 & +.2 & \cdot \\ . & 1.1 & +.2 \\ . & + & + \\ . & + & + \\ +.2 & + & 1.2 \\ + & \mathrm{r} & .\end{array}$

Además: (Ass., Al., Or., Cl.) 1. Sorbus aucuparia, +; Euphorbia hyberna, 1.1; Luzula silvatica subsp. henriquesii, 1,2; Osmunda regalis, 1.2; 2. Crataegus monogyna, 1.1; Castanea sativa, +; Tamus comunis, 1.1; Lathyrus montanus, +; Euphorbia amygdaloides, +; Hyacinthoides noncripta, +; Polygonatum odoratum, +; 3. Pyrus pyraster, 1.1; Frangula alnus, +; Saxifraga spathularis, +; Hypericum pulchrum, +; Crepis lampsanoides, +; Teucrium scorodonia, + .

Además: (Dif. de Fagetalia), 1. Woodwardia radicans, 2.3; Cardamine impatiens, $+; 3$. Acer pseudoplatanus, +; Ulmus glabra, +; Circaea lutetiana, 1.1; Milium effussum, 1.1; Lysimachia nemorum, +

Además: 1. Melittis melissophyllum, +; Ajuga reptans, 1.1; 2 . Angelica razulii, +; Laurus nobilis, r; Aquilegia vulgaris, r; Salix atrocinerea, +; 3. Geranium robertianum, +; Agrostis tenuis, +; Senecio nemorensis subsp. bayonensis, +; Lastraea limbosperma, + .

Localidades: 1. Lu: Trabada, Vilapena. 29TPJ4309. 2. C: Cerdido, devesa del Mera c. Casares, 29TNJ8826 3. Lu: Mondoñedo, devesa del Puerto de Campo do Oso. 29TPJ36605 
TABLA 3

Blechno spicantis-Quercetum roboris "facies de Castanea sativa"

№ de orden

Altitud (en Dm) $\quad 23 \quad 36 \quad 28 \quad 31$

$\begin{array}{llll}1 & 2 & 3 & 4\end{array}$

Cobertura (\%E1) $90 \quad 95 \quad 100 \quad 95$

Cobertura (\%E2) $\quad 90 \quad 90 \quad 75 \quad 90$

Inclinación $\quad 35^{\circ} \quad 30^{\circ} \quad 45^{\circ} \quad 35^{\circ}$

$\begin{array}{lllll}\text { Orientación } & \text { N } & \text { N } & \text { E } & \text { NW }\end{array}$

$\begin{array}{lllll}\text { Area (en m2) } & 180 & 150 & 250 & 100\end{array}$

№ de especies

$\begin{array}{llll}16 & 15 & 21 & 25\end{array}$

Características de Ass., Al., Or.

Castanea sativa

$\begin{array}{llll}5.4 & 4 . .4 & 5.5 & 4.4\end{array}$

Quercus robur

$\begin{array}{llll}1.1 & 2.2 & 1.1 & 1.1\end{array}$

Holcus mollis

$\begin{array}{llll}4.3 & 4.3 & 2.3 & 3.3\end{array}$

Blechnum spicant

$2.3+2.22 .2$

Hypericum pulchrum

$+\quad+1.1+$

Betula celtiberica

$\begin{array}{lll}2.1 & 1.1 \quad 2.2\end{array}$

Vaccinium myrtillus

$\begin{array}{llll}.+2 & 1.2+ & + & \\ + & +.2\end{array}$

Teucrium scorodonia

Melampyrum pratense

Physospermum cornubiense

Saxifraga spathularis

Omphalodes nitida

Características de Clase

Hedera helix

$\begin{array}{lll}1.2 \quad & 1.2 \quad 2.2\end{array}$

Dryopteris affinis

Frangula alnus

Stellaria holostea

Euphorbia dulcis

Lonicera periclymenum

Ilex aquifolium

Viola riviniana

\section{Compañeras}

Pteridium aquilinum

Erica arborea

Rubus gr. corylifolius

Arenaria montana

Daboecia cantabrica

Ruscus aculeatus

Arbutus unedo

Laurus nobilis

Polypodium interjectum

Agrostis tenuis

Festuca gr. ovina

Pseudoarrhenatherum longifc

Rumex acetosa

Anthoxanthum odoratum

Brachypodium pinnatum

Agrostis curtisii

Ulex gallii

Linaria triornithophora

$$
\begin{array}{cccc}
1.1 & + & \cdot & 1.1 \\
\cdot & 1.1 & + & \cdot \\
+.2 & \cdot & \cdot & +.2 \\
\cdot & \cdot & \mathrm{r} & 1.1 \\
\cdot & \cdot & + & 1.1 \\
\cdot & \cdot & + & \cdot \\
\cdot & \cdot & + & \cdot \\
& \cdot & & \\
1.1 & 2.3 & 3.2 & 1.1 \\
+ & 1.1 & \cdot & 1.1 \\
2.2 & 3.2 & \cdot & 3.3 \\
+ & \cdot & \cdot & 1.1 \\
+ & \cdot & + & \cdot \\
\cdot & \cdot & + & + \\
\cdot & 1.1 & \cdot & \cdot \\
\cdot & \cdot & \cdot & + \\
\cdot & \cdot & \cdot & + \\
2.1 & \cdot & \cdot & \cdot \\
\cdot & \cdot & 2.2 & \cdot \\
\cdot & \cdot & +.2 & \cdot \\
\cdot & \cdot & \cdot & + \\
\cdot & \cdot & + & \cdot \\
\cdot & \cdot & \cdot & 1.2 \\
\cdot & \cdot & + & \cdot \\
\cdot & \cdot & \cdot & +.2
\end{array}
$$

Localidades: 1. Lu: Mondoñedo, Lindín. 29TPJ35507. 2. Lu: Pontenova, Villaoruz. 29TPJ4504. 3. O: Taramundi, Esquío. 29TPJ5401. 4. Lu: Riotorto, Santadriao c. Galegos. 29TPH4594. 
TABLA 4.- Ulici europaei-Cytisetum ingramii Rivas-Martinez 1978

\begin{tabular}{|c|c|c|c|c|c|c|c|c|c|c|}
\hline $\mathrm{N} e$ de orden & 1 & 2 & 3 & 4 & 5 & 6 & 7 & 8 & 9 & 10 \\
\hline Altitud (en Dm) & - & - & 31 & 34 & - & 25 & 9 & 25 & 29 & - \\
\hline Cobertura $(\%)$ & 100 & 80 & 100 & 100 & 100 & 100 & 95 & 100 & 100 & 100 \\
\hline Inclinación & $15^{\circ}$ & $15^{\circ}$ & - & 50 & - & - & - & - & 109 & \\
\hline Orientación & $\mathrm{N}$ & $\mathrm{N}$ & - & SW & - & NW & - & NW & NE & \\
\hline Area $\left(e n m^{2}\right)$ & 60 & 80 & 80 & 20 & 60 & 80 & 30 & 30 & 80 & 70 \\
\hline $\mathrm{N} 2$ de especies & 9 & 11 & 12 & 12 & 10 & 12 & 14 & 17 & 17 & \\
\hline
\end{tabular}

\section{Características de asociación}

y unidades superiores

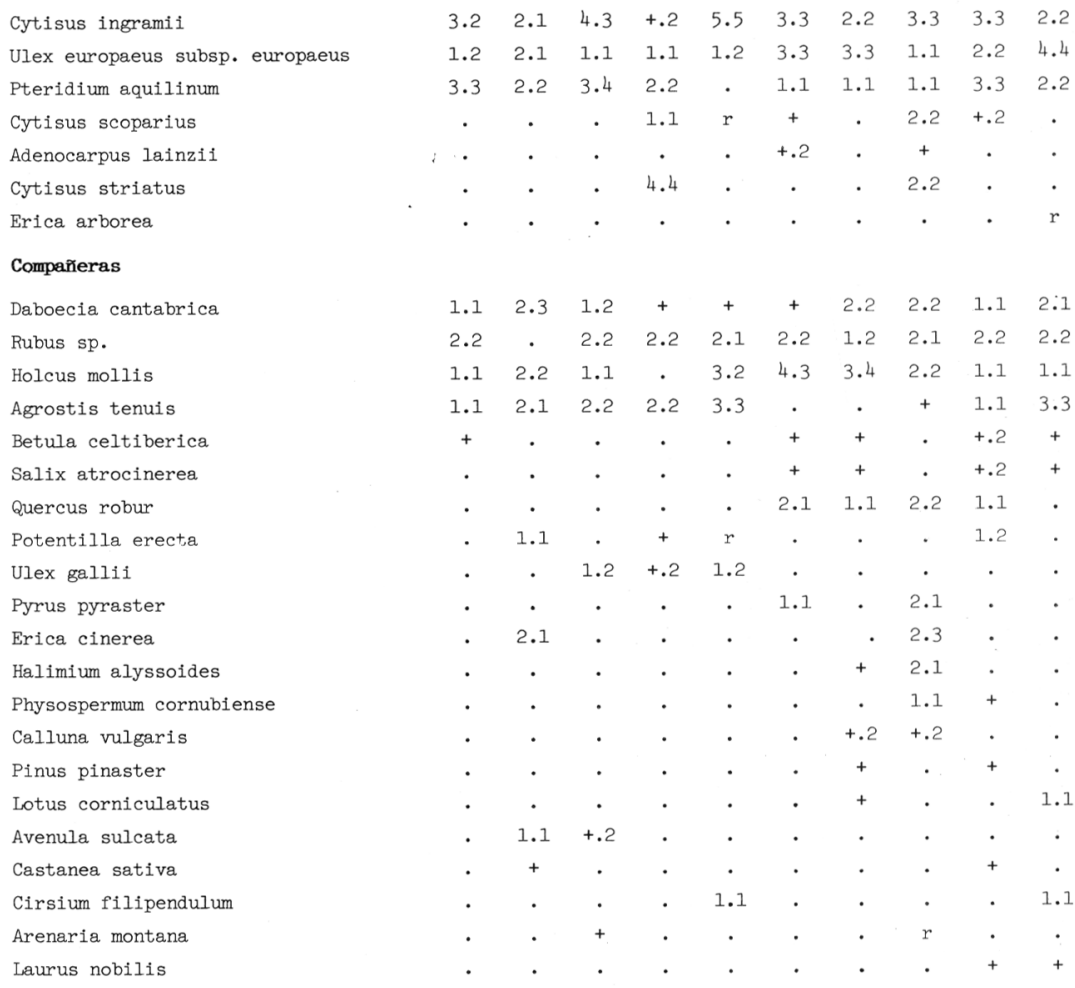

\section{Además:}

1. Asphodelus albus, +; 2. Hypericum pulchrum, $r$; 3. Cruciata glabra, 1.1; Lithodora prostrata, +; 4. Dactylis glomerata, +; Clinopodium vulgare, +; 7. Quercus pyrenaica, +; 9. Hedera helix, +; 10. Viola riviniana, 1.1; Brachypodium pinnatum subsp. rupestre, +.2; Hyacinthoides non-scripta, +.

\section{Localidades}
1. C: Campo de Hospital, c. Puente Neda.
6. C: De Neda a Bouzarredonda, río Castro.
2. C: Misma localidad que la anterior.
7. C: S. Saturnino.
3. C: Mosende.
8. C: De Neda a Bouzarredonda.
4. C: Casanova, ladera del Sor.
9. $\mathrm{C}$ : Devesos
5. C: As Neves, de As Pontes a Ortigueira.
10. C: De Insua a Casares. 


\title{
SOBRE LOS ROBLEDALES DEL LLANO DE LA SELVA (GERONA)
}

\author{
Luis VILAR \& Xavier VIÑAS
}

RESUMEN: El Llano de la Selva (Gerona) es una depresión que presenta condiciones climáticas y geológicas especiales que favorecen al bosque caducifolio frente al perennifolio de las Sierras vecinas. Se trata de robledales de Quercus pubescens de los cuales se describe una nueva subasociación.

Palabras clave: Fitosociología, Bosques, Robledales de Q. gr. pubescens, La Selva (Cataluña), Quercion ilicis, Quercion robori-petraeae..

SUMMARY: Two oak groves from Selva country (NE part of Iberian Peninsula) has been studied. The central part of this country (Selva Depression) have special geological and climatical characteristics which favour decidous species instead evergreen ones, which are common in the neighbouring mountains.

Key words: Phytosociology, Forests, Oak groves of Q.gr. pubescens, Quercion ilicis, Quercion robori-petraeae.

\section{N T R O D U C C I O N}

Algunas llanuras aluviales de la cuenca mediterránea, mayoritariamente zonas de cultivo en la actualidad, debían de asentar frondosos y espesos bosques en los cuales las especies caducifolias tendrían un papel principal respecto a las perennifolias. El hecho es conocido de diversos puntos: el Languedoc francés (Kielhauser, 1939 y Braun-Blanquet et al., 1952), el Mediterráneo Oriental (Quèzel, 1985)... En algunos puntos de la provincia de Gerona los bosques de llano aluvial son aún importantes en el paisaje y su estudio, a la vez que contribuye a la caracterización de la vegetación potencial del territorio, aporta nuevos datos para establecer el papel del roble y otras especies caducifolias en suelos sedimentarios mediterráneos.

Situación. La Depresión de la Selva se sitúa en el extremo septentrional de las Sierras Costeras Catalanas, desde la ciudad de Girona hasta el río Tordera, en el límite con la provincia de Barcelona (sector Vallesano-Empordanés). Geomorfológicamente, constituye un bloque deprimido de unos $200 \mathrm{Km}^{2}$ de superficie, entre 70 y 200 metros de altitud, rodeado de otros bloques elevados, todos ellos de naturaleza granítica y 